\title{
A DELAY REACTION-DIFFUSION MODEL OF THE SPREAD OF BACTERIOPHAGE INFECTION*
}

\author{
STEPHEN A. GOURLEY ${ }^{\dagger}$ AND YANG KUANG K $^{\ddagger}$
}

\begin{abstract}
This paper is a continuation of recent attempts to understand, via mathematical modeling, the dynamics of marine bacteriophage infections. Previous authors have proposed systems of ordinary differential delay equations with delay dependent coefficients. In this paper we continue these studies in two respects. First, we show that the dynamics is sensitive to the phage mortality function, and in particular to the parameter we use to measure the density dependent phage mortality rate. Second, we incorporate spatial effects by deriving, in one spatial dimension, a delay reactiondiffusion model in which the delay term is rigorously derived by solving a von Foerster equation. Using this model, we formally compute the speed at which the viral infection spreads through the domain and investigate how this speed depends on the system parameters. Numerical simulations suggest that the minimum speed according to linear theory is the asymptotic speed of propagation.
\end{abstract}

Key words. reaction-diffusion, delay, stage structure, through-stage death rate, traveling wave

AMS subject classifications. 92D25, 35K57, 35R10

DOI. $10.1137 /$ S0036139903436613

1. Introduction. It is known that bacteriophage infection can be a significant mechanism of mortality in marine prokaryotes (Bergh et al. [6], Proctor and Fuhrman [16]). These mortality mechanisms are critical in understanding the marine production processes. The constituents released by cell lysis can be an important pathway of nutrient recycling. This has direct bearing on issues such as global warming and topics of geochemical cycles. Viral infection also has direct implications for genetic exchange in the sea (Lenski and Levin [14], Bohannan and Lenski [7]).

Although we do not yet have a good understanding of the temporal or spatial scales at which host-virus encounters occur, it is clear that viral mortality must be explicitly considered in most models of the marine system. A case in point, recent experimental work suggests that the contamination of algal cells by viruses can serve as a regulatory mechanism in its bloom dynamics. Beltrami and Carroll [1] formulated a simple trophic model including virus-induced mortality. Their model succeeded in mimicking the actual algal bloom patterns of several species.

Our main interest in this paper is to explore how viral mortality affects both the temporal and spatial dynamics of marine bacteria and cyanobacteria. Recently, Beretta and Kuang [4] formulated and carried out a detailed study of the temporal viral-bacteria model

$$
\begin{aligned}
& \frac{d S}{d t}=\alpha S(t)\left(1-\frac{S(t)+I(t)}{C}\right)-K S(t) P(t), \\
& \frac{d I}{d t}=-\mu_{i} I(t)+K S(t) P(t)-e^{-\mu_{i} T} K S(t-T) P(t-T), \\
& \frac{d P}{d t}=\beta-\mu_{p} P(t)-K S(t) P(t)+b e^{-\mu_{i} T} K S(t-T) P(t-T) .
\end{aligned}
$$

*Received by the editors October 24, 2003; accepted for publication (in revised form) May 17, 2004; published electronically January 5, 2005.

http://www.siam.org/journals/siap/65-2/43661.html

${ }^{\dagger}$ Corresponding author. Department of Mathematics and Statistics, University of Surrey, Guildford, Surrey GU2 7XH, UK (s.gourley@surrey.ac.uk). Correspondence should be directed to this author.

${ }^{\ddagger}$ Department of Mathematics and Statistics, Arizona State University, Tempe, AZ 85287 (kuang@ asu.edu). The research of this author was partially supported by NSF grant DMS-0077790. 
This system of delay differential equations models a population of marine bacteria in which the individuals are subject to infection by viruses, also known as bacteriophages. Prior to that, these authors (Beretta and Kuang [2]) modeled and studied the same process by a set of nonlinear ordinary differential equations, and Carletti [10] has studied the stochastic extension of that model. In system (1.1), $S$ is the density (i.e., number of bacteria per liter) of susceptible bacteria, $I$ is the density of infected bacteria, and $P$ is the density (number of viruses per liter) of viruses (phages). Viruses $P$ attack the susceptible bacteria $S$, and a bacterium becomes infected $I$ when a virus successfully injects itself through the bacterial membrane. The virus then starts replicating inside the bacterium, and then all the bacterium's resources are directed to replication of the virus. The infected bacterium does not replicate itself by division; only susceptible bacteria are capable of doing so. After a latency time $T$, an infected bacterium will die by lysis; i.e., the bacterium explodes releasing $b$ copies $(b>1)$ of the virus into the solution, which are then free to attack other susceptible bacteria. An infected bacterium may die other than by viral lysis; we allow for this by the term $-\mu_{i} I(t)$. The differential equation for $I(t)$ is derived from the fact that $I(t)$ is given by

$$
I(t)=\int_{0}^{T} e^{-\mu_{i} \tau} K S(t-\tau) P(t-\tau) d \tau
$$

which expresses the fact that the number of recruits into the infected class between times $t-(\tau+d \tau)$ and $t-\tau$ is $K S(t-\tau) P(t-\tau) d \tau$, the number of these still alive at time $t$ is obtained by multiplying by $e^{-\mu_{i} \tau}$, and then the integral totals up the contributions from all relevant previous times, i.e., up to $T$ time units ago.

In the virus equation, the third equation of (1.1), all mortalities of viruses are accounted for by the term $-\mu_{p} P(t)$. The $\beta$ term, where $\beta>0$, models a constant inflow of phages from outside the system. In the absence of viruses the bacteria grow logistically. The rate of infection is given by the law of mass action to be $K S(t) P(t)$.

Beretta and Kuang [4] assumed that infected bacteria still compete with susceptible bacteria for common resources. This is represented by the $-(S+I) / C$ term in the first equation of (1.1). This is clearly a disputed subject. For example, a model by Campbell [8] consists of the following equations:

$$
\begin{aligned}
& \frac{d S(t)}{d t}=\alpha S(t)\left(1-\frac{S(t)}{C}\right)-K S(t) P(t), \\
& \frac{d P(t)}{d t}=b K S(t-T) P(t-T)-\mu_{p} P(t)-K S(t) P(t),
\end{aligned}
$$

where

$$
I(t)=\int_{t-T}^{t} K S(\theta) P(\theta) d \theta
$$

Clearly, in (1.3) the competition for common resources and additional mortality rate endured by infected bacteria is neglected. The equations (1.3), (1.4) can be obtained from (1.1) by setting $\beta=0, \mu_{i}=0$. Extensions of the above Campbell model can be found in Beretta, Carletti, and Solimano [3] (taking into account environmental fluctuations) and Carletti [9] (replacing $b$ by $b e^{-\mu_{i} T}$ ).

In the present paper, like the model of Campbell [8], we assume that once a bacterium becomes infected by a virus, it no longer competes with susceptibles for 
resources. We will allow the possibility of a density dependent mortality term in the phage equation. In Beretta and Kuang [4], and also in the present paper, it is assumed that $T$ and $b$ are constant and the same for the whole population. Modifications of this assumption (e.g., replacing the constant incubation time $T$ by a distribution of incubation times modeled using a probability density function) are the subject of further work presently in progress.

In the next section, we will present our delay model of bacteriophage infection and a simple preliminary result on the positivity of its solutions. This is followed by a short section on the global stability of the disease-free equilibrium. The analysis of endemic equilibrium is highly nontrivial and we provide only generic conditions for its stability switch. To complement this analytic work, we present some carefully designed and data-based simulation results. We then proceed to formulate and study a delay reaction-diffusion model of the spread of bacteriophage infection. The paper ends with a discussion.

2. Preliminaries. Most of our effort will be devoted to understanding the system

$$
\begin{aligned}
& S^{\prime}(t)=\alpha S(t)\left(1-\frac{S(t)}{\gamma}\right)-K S(t) P(t) \\
& P^{\prime}(t)=-\mu_{p} P(t)-m P^{2}(t)-K S(t) P(t)+b K e^{-\mu_{i} T} S(t-T) P(t-T),
\end{aligned}
$$

and with a reaction-diffusion version of (2.1). The initial conditions for (2.1) are

$$
\begin{aligned}
& S(s)=S^{0}(s) \geq 0, s \in[-T, 0], \quad \text { with } S^{0}(0)>0, \\
& P(s)=P^{0}(s) \geq 0, s \in[-T, 0], \quad \text { with } P^{0}(0)>0,
\end{aligned}
$$

where $S^{0}$ and $P^{0}$ are prescribed continuous functions. Our system (2.1) differs from that studied in [4] in three respects: (i) we do not have an inflow of phages from outside the system, (ii) we allow the possibility of a density dependent mortality term (the term $-m P^{2}$ in (2.1)), and (iii) we assume that an infected bacterium no longer competes with the susceptibles for resources. The latter assumption means that we do not need the differential equation for $I(t)$ for the analysis (though $I(t)$ is still given by (1.2)). An additional difference is that in the present paper we shall consider the effects of including diffusion to model the motion of the phages and bacteria.

If we had $P^{0}(s) \equiv 0$ on $[-T, 0]$, the method of steps would immediately yield $P(t)=0$ for all $t>0$. The dynamics of $S(t)$ would then be governed by the logistic equation. Similarly, if $S^{0}(s) \equiv 0$, then clearly $S(t)$ remains zero for all $t>0$ and thus $P(t) \rightarrow 0$ as $t \rightarrow \infty$. These trivial cases are removed from consideration by the assumptions in (2.2).

Proposition 1. Solutions of (2.1), (2.2) satisfy $S(t)>0, P(t)>0$ for all $t>0$.

Proof. The equation for $S(t)$ in (2.1) contains a factor of $S(t)$ and therefore positivity for $S(t)$ follows by the standard argument. For $P(t)$, note that on $t \in[0, T]$ we have $P^{\prime}(t) \geq-\mu_{p} P(t)-m P^{2}(t)-K S(t) P(t)$ so that $P(t) \geq \tilde{P}(t)$, where $\tilde{P}$ is the solution of $\tilde{P}^{\prime}(t)=-\mu_{p} \tilde{P}(t)-m \tilde{P}^{2}(t)-K S(t) \tilde{P}(t)$ satisfying $\tilde{P}(0)=P(0)>0$. Clearly $\tilde{P}(t)>0$ for all $t>0$, and so we conclude that $P(t)>0$ for all $t>0$. The proof is complete.

3. Equilibria and their stability. The equilibria of $(2.1)$ are $(S, P)=(0,0)$, the disease-free equilibrium $(\gamma, 0)$, and possibly an endemic equilibrium

$$
\left(S^{*}, P^{*}\right):=\left(\frac{m \gamma \alpha+K \gamma \mu_{p}}{m \alpha+K^{2} \gamma\left(b e^{-\mu_{i} T}-1\right)}, \frac{\alpha \gamma K\left(b e^{-\mu_{i} T}-1\right)-\alpha \mu_{p}}{m \alpha+K^{2} \gamma\left(b e^{-\mu_{i} T}-1\right)}\right) .
$$


The latter is ecologically relevant if and only if

$$
b e^{-\mu_{i} T}>1+\frac{\mu_{p}}{\gamma K},
$$

which, of course, can only possibly hold for $T$ up to a finite value. As long as (3.2) holds, there is an endemic equilibrium. Note that as $m \rightarrow \infty$ the endemic equilibrium approaches the disease-free equilibrium $(\gamma, 0)$.

We shall first prove that, if condition (3.2) does not hold, then any positive solution approaches the disease-free equilibrium $(\gamma, 0)$.

Theorem 1. Assume that

$$
b e^{-\mu_{i} T} \leq 1+\frac{\mu_{p}}{\gamma K}
$$

Then any solution of (2.1),(2.2) satisfies

$$
\lim _{t \rightarrow \infty}(S(t), P(t))=(\gamma, 0) .
$$

Proof. Consider the positive definite functional

$$
V=S-\gamma-\gamma \ln \frac{S}{\gamma}+\frac{\gamma K}{\mu_{p}} P+\frac{b \gamma K^{2}}{\mu_{p}} e^{-\mu_{i} T} \int_{t-T}^{t} S(s) P(s) d s .
$$

Differentiating along solutions of (2.1) yields

$$
\begin{aligned}
V^{\prime} & =-\frac{\alpha}{\gamma}(S-\gamma)^{2}-\frac{\gamma m K}{\mu_{p}} P^{2}+K\left(\frac{b \gamma K}{\mu_{p}} e^{-\mu_{i} T}-\frac{\gamma K}{\mu_{p}}-1\right) S P \\
& \leq-\frac{\alpha}{\gamma}(S-\gamma)^{2} .
\end{aligned}
$$

Thus

$$
V(t)+\frac{\alpha}{\gamma} \int_{0}^{t}(S(s)-\gamma)^{2} d s \leq V(0),
$$

and, letting $t \rightarrow \infty$, we conclude that $|S(t)-\gamma| \in L^{2}(0, \infty)$ so that $S(t) \rightarrow \gamma$ as $t \rightarrow \infty$. The differential equations (2.1) then yield $P(t) \rightarrow 0$. The proof is complete.

3.1. The endemic equilibrium: Linearized analysis. Let us investigate the endemic equilibrium $\left(S^{*}, P^{*}\right)$ given by (3.1). In this subsection we shall assume, of course, that (3.2) holds, so that the equilibrium is feasible. The linearized analysis about the endemic equilibrium is algebraically quite complicated. The main reason for this is that the delay $T$ appears not only in the $S(t-T) P(t-T)$ term in the second equation of (2.1), but also in the factor $e^{-\mu_{i} T}$ in front of that term. The paper by Wolkowicz, Xia, and Wu [20] shows how such additional factors involving time delay can appear in distributed delay equations. Surprisingly, this represents a significant complication and prevents us from analytically computing the precise parameter regimes in which the endemic equilibrium can change stability as the delay $T$ is increased, or the actual values of $T$ when stability switches occur. Note further that the equilibrium itself depends on $T$ and exists only for $T$ up to a finite value. This renders many of the existing stability switch methods (see Kuang [12]) powerless. However, a method has recently been developed by Beretta and Kuang [5] to address the problem of computing stability switches for delay equations which do not lend 
themselves to classical methods because of these complications. We shall use this method in this section.

To linearize about $\left(S^{*}, P^{*}\right)$ we set $S=S^{*}+\tilde{S}$ and $P=P^{*}+\tilde{P}$. Ignoring higher order terms in $\tilde{S}, \tilde{P}$ gives us the linearized system

$$
\begin{aligned}
\tilde{S}^{\prime}(t)= & -\frac{\alpha}{\gamma} S^{*} \tilde{S}(t)-K S^{*} \tilde{P}(t), \\
\tilde{P}^{\prime}(t)= & -K P^{*} \tilde{S}(t)-\left(\mu_{p}+2 m P^{*}+K S^{*}\right) \tilde{P}(t) \\
& +b K e^{-\mu_{i} T}\left(P^{*} \tilde{S}(t-T)+S^{*} \tilde{P}(t-T)\right) .
\end{aligned}
$$

We shall find it convenient to introduce the parameter

$$
\rho_{T}=\frac{\gamma K}{\mu_{p}}\left(b e^{-\mu_{i} T}-1\right) .
$$

Then the endemic equilibrium $\left(S^{*}, P^{*}\right)$ exists if and only if

$$
\rho_{T}>1 \text {. }
$$

In terms of $\rho_{T}$,

$$
\left(S^{*}, P^{*}\right)=\left(\frac{\gamma\left(m \alpha+K \mu_{p}\right)}{m \alpha+K \mu_{p} \rho_{T}}, \frac{\alpha \mu_{p}\left(\rho_{T}-1\right)}{m \alpha+K \mu_{p} \rho_{T}}\right) .
$$

Nontrivial solutions of the linearized system of the form $(\tilde{S}(t), \tilde{P}(t))=e^{\lambda t}\left(c_{1}, c_{2}\right)$ exist if and only if

$$
D(\lambda ; T)=0
$$

where

$$
D(\lambda ; T)=\lambda^{2}+a(T) \lambda+b(T) \lambda e^{-\lambda T}+c(T)+d(T) e^{-\lambda T}
$$

and

$$
\begin{aligned}
& a(T)=\frac{\alpha\left(m \alpha+K \mu_{p}\right)+m \alpha\left(K \gamma+2 \mu_{p} \rho_{T}-\mu_{p}\right)+K \mu_{p}\left(K \gamma+\mu_{p} \rho_{T}\right)}{m \alpha+K \mu_{p} \rho_{T}} \\
& b(T)=-\frac{b \gamma K e^{-\mu_{i} T}\left(m \alpha+K \mu_{p}\right)}{m \alpha+K \mu_{p} \rho_{T}} \\
& c(T)=\frac{\alpha\left(m \alpha+K \mu_{p}\right)\left\{m \alpha\left(K \gamma+\left(2 \rho_{T}-1\right) \mu_{p}\right)+K \mu_{p}\left(\mu_{p} \rho_{T}+\left(2-\rho_{T}\right) K \gamma\right)\right\}}{\left(m \alpha+K \mu_{p} \rho_{T}\right)^{2}} \\
& d(T)=\frac{b K \gamma \alpha e^{-\mu_{i} T}\left(m \alpha+K \mu_{p}\right)\left\{K \mu_{p}\left(\rho_{T}-2\right)-m \alpha\right\}}{\left(m \alpha+K \mu_{p} \rho_{T}\right)^{2}}
\end{aligned}
$$

Keeping in mind that $b>1$, it is straightforward to see that when $T=0$ the equilibrium $\left(S^{*}, P^{*}\right)$, if feasible, is linearly stable. This is because when $T=0,(3.5)$ becomes a quadratic in $\lambda$, and it is easy to see that $a(0)+b(0)>0$ and $c(0)+d(0)>0$. The question is whether the equilibrium can undergo any stability switch as $T$ is increased, remembering that the equilibrium is only feasible up to a finite value of $T$. To identify a stability switch we seek solutions of the characteristic equation $D(\lambda ; T)=0$ of the 
form $\lambda= \pm i \omega$, with $\omega$ a real positive number. We find that it is necessary for $\omega$ to satisfy

$$
\omega^{4}+\left(a^{2}(T)-2 c(T)-b^{2}(T)\right) \omega^{2}+c^{2}(T)-d^{2}(T)=0 .
$$

However, the existence for a particular $T$ of a real root $\omega(T)$ of (3.10) does not in itself imply that a stability switch occurs at that value of $T$, since $T$ also has to satisfy (3.11) and (3.12) below. Nonetheless, certain general analytical conclusions can be drawn in spite of the algebra. Straightforward but tedious computations show that, for any parameter values consistent with $\rho_{T}>1$ (i.e., with existence of the endemic equilibrium $\left(S^{*}, P^{*}\right)$ ), we have

$$
a^{2}(T)-2 c(T)-b^{2}(T)>0 .
$$

In light of this fact, and assuming that $\left(S^{*}, P^{*}\right)$ is feasible when $T=0$, certain conclusions follow.

(i) A stability switch cannot occur in an interval of $T$ throughout which $c^{2}(T)>$ $d^{2}(T)$.

(ii) If there are values of $T$ with $c^{2}(T)<d^{2}(T)$, then a stability switch may occur as $T$ is varied. Pairs of eigenvalues cross the imaginary axis as $T$ passes through certain critical values. The critical values of $T$ and the corresponding purely imaginary eigenvalues $\pm i \omega(T), \omega(T)>0$, are given implicitly by

$$
\begin{gathered}
\sin (\omega(T) T)=\frac{b(T) \omega(T)\left(\omega^{2}(T)-c(T)\right)+\omega(T) a(T) d(T)}{\omega^{2}(T) b^{2}(T)+d^{2}(T)}, \\
\cos (\omega(T) T)=\frac{d(T)\left(\omega^{2}(T)-c(T)\right)-\omega^{2}(T) a(T) b(T)}{\omega^{2}(T) b^{2}(T)+d^{2}(T)}, \\
\omega^{2}(T)=\frac{1}{2}\left(-a^{2}(T)+2 c(T)+b^{2}(T)\right. \\
\left.+\sqrt{a^{4}(T)-4 a^{2}(T) c(T)-2 a^{2}(T) b^{2}(T)+4 c(T) b^{2}(T)+b^{4}(T)+4 d^{2}(T)}\right),
\end{gathered}
$$

where $a(T), b(T), c(T)$, and $d(T)$ are given by (3.6), (3.7), (3.8), and (3.9) above. It is impossible to solve these equations for $T$ explicitly, so we shall use the procedure described in Beretta and Kuang [5]. According to this procedure, we define $\theta(T) \in$ $[0,2 \pi)$ such that $\sin \theta(T)$ and $\cos \theta(T)$ are given by the right-hand sides of (3.11) and (3.12), respectively, with $\omega(T)$ given by (3.13). This defines $\theta(T)$ in a form suitable for numerical evaluation using standard software. Then $T$ is given (still implicitly) by

$$
T=\frac{\theta(T)+2 n \pi}{\omega(T)}, \quad n=0,1,2, \ldots,
$$

and the idea is to identify the roots of this equation for various $n$, i.e., to solve numerically the equation $S_{n}(T)=0$ for $n=0,1,2$, where

$$
S_{n}(T)=T-\left(\frac{\theta(T)+2 n \pi}{\omega(T)}\right), \quad n=0,1,2, \ldots
$$


Accurate plots of these functions $S_{n}(T)$ quickly reveal whether stability switches can occur or not, but one must remember to keep track of the feasibility of the equilibrium $\left(S^{*}, P^{*}\right)$ since it disappears completely (by coalescing with the disease-free equilibrium $(\gamma, 0))$ at a finite value of the delay $T$.

By reference to (i) above, it is possible to obtain sufficient and easily verifiable conditions for the equilibrium $\left(S^{*}, P^{*}\right)$ to remain locally stable. Indeed, the condition $c^{2}(T)>d^{2}(T)$ amounts to

$$
\begin{gathered}
m^{2}\left\{\alpha^{2}\left(K \gamma+\left(2 \rho_{T}-1\right) \mu_{p}\right)^{2}-\alpha^{2} b^{2} K^{2} \gamma^{2} e^{-2 \mu_{i} T}\right\} \\
+2 \alpha K \mu_{p} m\left\{\left(K \gamma+\left(2 \rho_{T}-1\right) \mu_{p}\right)\left(\mu_{p} \rho_{T}+\left(2-\rho_{T}\right) K \gamma\right)+\left(\rho_{T}-2\right) b^{2} K^{2} \gamma^{2} e^{-2 \mu_{i} T}\right\} \\
+K^{2} \mu_{p}^{2}\left(\mu_{p} \rho_{T}+\left(2-\rho_{T}\right) K \gamma\right)^{2}-K^{2} \mu_{p}^{2}\left(\rho_{T}-2\right)^{2} b^{2} K^{2} \gamma^{2} e^{-2 \mu_{i} T}>0 .
\end{gathered}
$$

Thus, if (3.15) holds, then $\left(S^{*}, P^{*}\right)$, if feasible, is locally stable. The coefficient of $m^{2}$ in (3.15) is automatically positive if $\rho_{T}>1$ (the condition for feasibility of $\left(S^{*}, P^{*}\right)$ ), and therefore one parameter regime in which (3.15) is satisfied is that the parameter $m$ be large.

For the convenience of comparison and computation, we perform the same dimensionless analysis as was carried out in Beretta and Kuang [4]. We choose the dimensionless time as $\tau=K \gamma t$. Note that one unit of the dimensionless time scale, i.e., $\tau=1$, corresponds to $t_{\tau}=(1 / K \gamma)$ in the original time unit. We also need the dimensionless variables

$$
s=\frac{S}{\gamma}, \quad p=\frac{P}{\gamma} .
$$

Below are the dimensionless parameters:

$$
a=\frac{\alpha}{K \gamma}, \quad m_{p}=\frac{\mu_{p}}{K \gamma}, \quad m_{i}=\frac{\mu_{i}}{K \gamma}, \quad m_{q}=\frac{m}{K} .
$$

Equations (2.1) have the dimensionless form

$$
\left\{\begin{array}{l}
\frac{d s(\tau)}{d \tau}=a s(\tau)-a s^{2}(\tau)-s(\tau) p(\tau) \\
\frac{d p(\tau)}{d \tau}=-m_{p} p(\tau)-m_{q} p^{2}(\tau)-s(\tau) p(\tau)+b e^{-m_{i} T_{\tau}} s\left(\tau-T_{\tau}\right) p\left(\tau-T_{\tau}\right)
\end{array}\right.
$$

The values for the dimensionless parameters and the dimensionless time scale are taken from the model of Beretta and Kuang [4] (the original parameter estimates are due to Okubo). They are

$$
a=10, \quad m_{p}=14.925,
$$

with $t_{\tau}=(1 / K \gamma)=7.4627$ days and an average latency time $T \simeq 0.303$ days. We have no estimates for $m_{i}=\left(\mu_{i} / K \gamma\right)$, but it seems reasonable to assume it is smaller than $m$ (since the main cause of mortality is the lysis of infected cells). We assume $m_{i} \simeq 0.1 m_{p}$. In addition, we do not have an estimate on $m_{q}$. In the following computational work, we assume that $m_{q} \simeq 0.1$, a value close to zero. Figure 1 is the result of an application of the stability switch theory of Beretta and Kuang [5] for this set of parameters (except that we vary the latency period).

Figure 2 provides simulation results for the above set of parameters with four representative values of latency periods. Clearly Figure 2 confirms the findings embodied in Figure 1. 


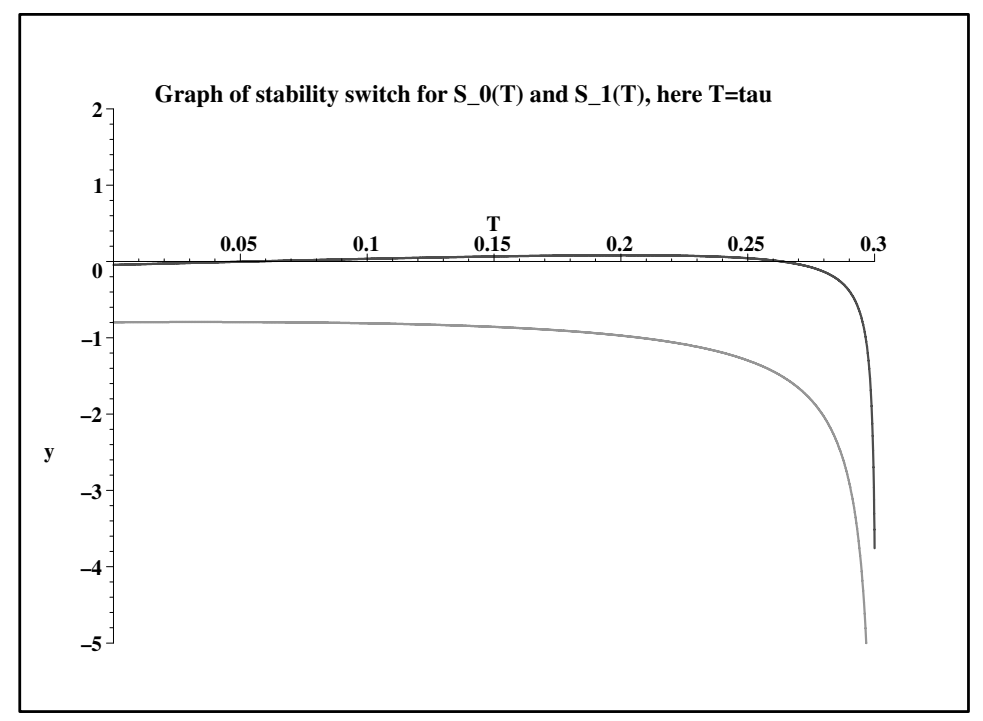

FIG. 1. Plots of the functions $S_{0}(\tau)$ (upper curve) and $S_{1}(\tau)$ (lower curve). Parameter values used are $\mu_{p}=14.925, b=75, \mu_{i}=1.5, \alpha=10$, and $m=0.1$. The equilibrium is feasible for $0 \leq \tau<\ln \left(b /\left(1+\mu_{p}\right)\right) / \mu_{i} \equiv \tau_{e} \approx 1.033$.
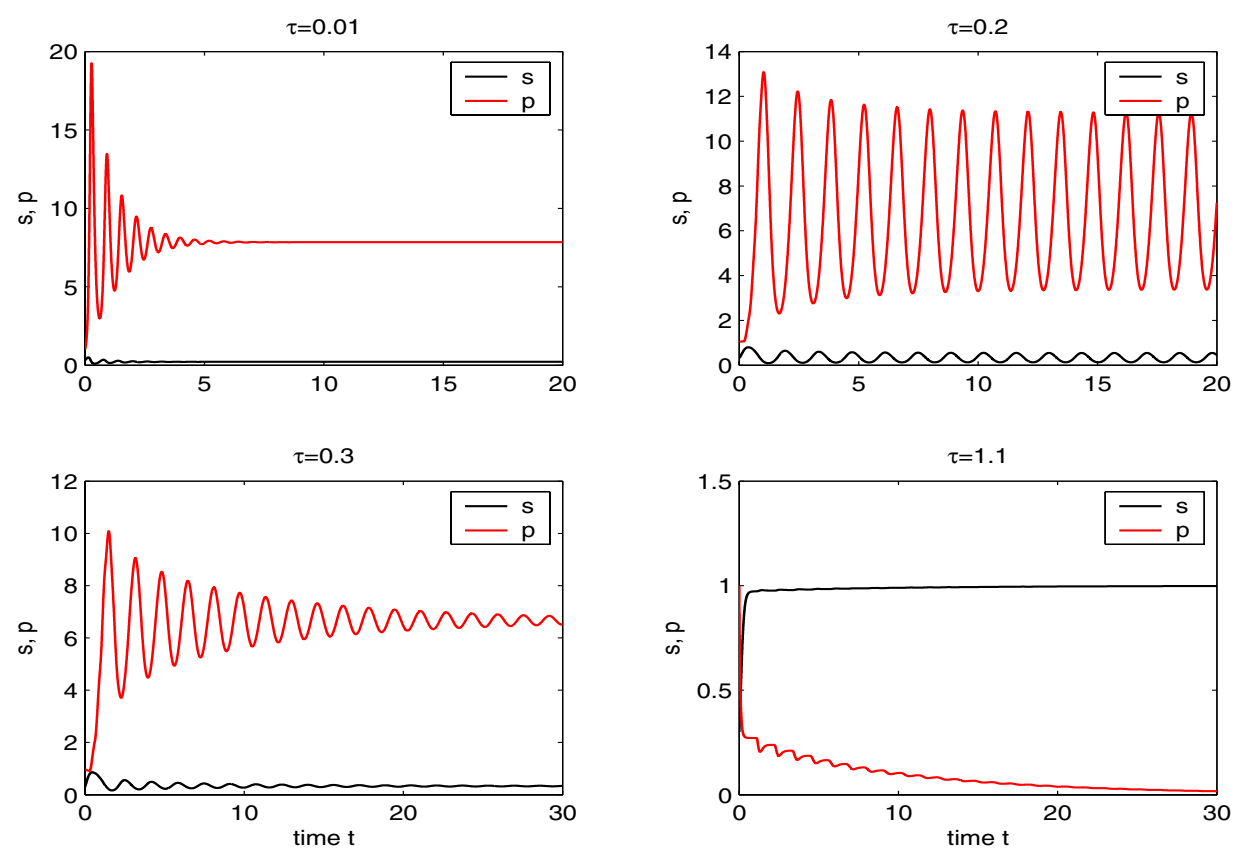

FIG. 2. A solution of model (3.16) with $s(\theta)=0.3, p(\theta)=1, \theta \in[-\tau, 0]$, where $\mu_{p}=14.925$, $b=75, \mu_{i}=1.5, \alpha=10, m=0.1$, and $\tau$ varies from 0.01 to 1.1 . 
4. Diffusive models. In this section we propose some reaction-diffusion extensions of system (2.1). The main issues here are (i) what types of diffusion are appropriate, and (ii) derivation of the time-delay terms for the case when there is diffusion. The latter point is important because infectives can move during the period between infection and lysis, so that when an infective dies by lysis it will release the $b$ copies of the virus into the water at a different location from where it originally became infected. We shall show how this can be accounted for in the modeling by including time and age as independent variables and using an age-structured model approach. The approach described here has also been used by many other investigators (see, e.g., Smith [17], So, Wu, and Zou [18], and Gourley and So [11]).

For the simplest case of Fickian diffusion, and working on an infinite one-dimensional domain $-\infty<x<\infty$, system (2.1) becomes

$$
\begin{aligned}
\frac{\partial S(x, t)}{\partial t}= & D_{s} \frac{\partial^{2} S(x, t)}{\partial x^{2}}+\alpha S(x, t)\left(1-\frac{S(x, t)}{\gamma}\right)-K S(x, t) P(x, t), \\
\frac{\partial P(x, t)}{\partial t}= & D_{p} \frac{\partial^{2} P(x, t)}{\partial x^{2}}-\mu_{p} P(x, t)-m P^{2}(x, t)-K S(x, t) P(x, t) \\
& +b \times\{\text { rate of death of infectives by lysis }\}
\end{aligned}
$$

where $D_{s}$ and $D_{p}$ are the diffusivities of the susceptibles and the phages. The last term in the $P$ equation reflects the fact that each time an infective dies by lysis, it releases $b$ copies of the virus, and we must now compute an expression for the term in curly brackets. As a first step in doing so, we shall indicate how to compute the density $I(x, t)$ of infectives at $(x, t)$. This will be achieved by using a standard agestructured model approach. Let $i(x, t, a)$ be the density of infectives at $(x, t)$ of age $a$. We assume that $i$ satisfies the von Foerster-type equation

$$
\frac{\partial i}{\partial t}+\frac{\partial i}{\partial a}=D_{i} \frac{\partial^{2} i}{\partial x^{2}}-\mu_{i} i
$$

where $D_{i}$ is the diffusivity of the infectives. The age of an infective will be measured from its time of infection so that, by the law of mass action,

$$
i(x, t, 0)=K S(x, t) P(x, t) .
$$

We want to solve (4.2) subject to (4.3) to obtain $i(x, t, a)$. The total density of infectives at $(x, t)$ will then be obtained by totaling all those of "age" less than $T$ (since older ones will have died by lysis); thus

$$
I(x, t)=\int_{0}^{T} i(x, t, a) d a .
$$

Expression (4.4) can then be used to find the rate of death of infectives by lysis which is required for model (4.1).

Let

$$
i^{r}(x, a)=i(x, a+r, a) .
$$

Then

$$
\frac{\partial i^{r}}{\partial a}=\left[\frac{\partial i}{\partial t}+\frac{\partial i}{\partial a}\right]_{t=a+r}=\left[D_{i} \frac{\partial^{2} i}{\partial x^{2}}-\mu_{i} i\right]_{t=a+r}
$$


so that

$$
\frac{\partial i^{r}}{\partial a}=D_{i} \frac{\partial^{2} i^{r}}{\partial x^{2}}-\mu_{i} i^{r}
$$

Applying the Fourier transform

$$
\widehat{i^{r}}(s, a)=\mathcal{F}\left\{i^{r}(x, a) ; x \rightarrow s\right\}=\int_{-\infty}^{\infty} i^{r}(x, a) e^{-i s x} d x
$$

to (4.5) gives

$$
\frac{\partial \widehat{i^{r}}(s, a)}{\partial a}=-\left(D_{i} s^{2}+\mu_{i}\right) \widehat{i^{r}}(s, a)
$$

the solution of which is

$$
\begin{aligned}
\widehat{i^{r}}(s, a) & =\widehat{i^{r}}(s, 0) e^{-\left(D_{i} s^{2}+\mu_{i}\right) a} \\
& =\mathcal{F}\{K S(x, r) P(x, r) ; x \rightarrow s\} e^{-\left(D_{i} s^{2}+\mu_{i}\right) a} \\
& =\mathcal{F}\{K S(x, r) P(x, r) ; x \rightarrow s\} \mathcal{F}\left\{\frac{e^{-\mu_{i} a}}{2 \sqrt{\pi D_{i} a}} e^{-x^{2} / 4 D_{i} a} ; x \rightarrow s\right\}
\end{aligned}
$$

since

$$
\widehat{i^{r}}(s, 0)=\mathcal{F}\{i(x, r, 0) ; x \rightarrow s\}=\mathcal{F}\{K S(x, r) P(x, r) ; x \rightarrow s\}
$$

and

$$
e^{-\left(D_{i} s^{2}+\mu_{i}\right) a}=\mathcal{F}\left\{\frac{e^{-\mu_{i} a}}{2 \sqrt{\pi D_{i} a}} e^{-x^{2} / 4 D_{i} a} ; x \rightarrow s\right\} .
$$

By the convolution theorem for Fourier transforms,

$$
i(x, a+r, a)=i^{r}(x, a)=\int_{-\infty}^{\infty} \frac{e^{-\mu_{i} a}}{2 \sqrt{\pi D_{i} a}} e^{-(x-y)^{2} / 4 D_{i} a} K S(y, r) P(y, r) d y .
$$

Hence

$$
i(x, t, a)=\int_{-\infty}^{\infty} \frac{e^{-\mu_{i} a}}{2 \sqrt{\pi D_{i} a}} e^{-(x-y)^{2} / 4 D_{i} a} K S(y, t-a) P(y, t-a) d y
$$

and so

$$
I(x, t)=\int_{0}^{T} \int_{-\infty}^{\infty} \frac{e^{-\mu_{i} a}}{2 \sqrt{\pi D_{i} a}} e^{-(x-y)^{2} / 4 D_{i} a} K S(y, t-a) P(y, t-a) d y d a
$$

or, after the substitution $a=t-\tau$,

$$
I(x, t)=\int_{t-T}^{t} \int_{-\infty}^{\infty} \frac{e^{-\mu_{i}(t-\tau)}}{2 \sqrt{\pi D_{i}(t-\tau)}} e^{-(x-y)^{2} / 4 D_{i}(t-\tau)} K S(y, \tau) P(y, \tau) d y d \tau .
$$

From this, we see that $I(x, t)$ obeys

$$
\begin{aligned}
\frac{\partial I(x, t)}{\partial t}= & D_{i} \frac{\partial^{2} I(x, t)}{\partial x^{2}}-\mu_{i} I(x, t)+K S(x, t) P(x, t) \\
& -K e^{-\mu_{i} T} \int_{-\infty}^{\infty} \frac{e^{-(x-y)^{2} / 4 D_{i} T}}{2 \sqrt{\pi D_{i} T}} S(y, t-T) P(y, t-T) d y
\end{aligned}
$$


and it is clear that the last term of this is the rate of death of infectives by lysis. Thus, system (4.1) becomes

$$
\begin{aligned}
\frac{\partial S(x, t)}{\partial t}= & D_{s} \frac{\partial^{2} S(x, t)}{\partial x^{2}}+\alpha S(x, t)\left(1-\frac{S(x, t)}{\gamma}\right)-K S(x, t) P(x, t) \\
\frac{\partial P(x, t)}{\partial t}= & D_{p} \frac{\partial^{2} P(x, t)}{\partial x^{2}}-\mu_{p} P(x, t)-m P^{2}(x, t)-K S(x, t) P(x, t) \\
& +b K e^{-\mu_{i} T} \int_{-\infty}^{\infty} \frac{e^{-(x-y)^{2} / 4 D_{i} T}}{2 \sqrt{\pi D_{i} T}} S(y, t-T) P(y, t-T) d y
\end{aligned}
$$

The formulation of a simple reaction-diffusion extension of (2.1) is complete. Like (2.1), system (4.6) does not involve the infectives $I(x, t)$ directly, but it does involve the parameter $D_{i}$ which measures their diffusivity.

System (4.6) is to be solved on the domain $-\infty<x<\infty$. Reaction-diffusion systems with delay are quite difficult to study, and in this paper we will not attempt a systematic study of all the dynamics of (4.6). It is of interest to investigate what (4.6) tells us about the spatial spread of a virus infection in a population of bacteria. Mathematically, it is therefore reasonable to look for traveling wave solutions of (4.6) connecting the disease-free equilibrium $(\gamma, 0)$ with the endemic equilibrium $\left(S^{*}, P^{*}\right)$ given by (3.1), assuming (3.2) holds so that an endemic equilibrium exists. A traveling front solution connecting these equilibria can model an invasion by the virus into the domain.

A traveling wave solution is one that travels at a constant speed $c$ without changing shape. Mathematically, it is a solution that depends on $x$ and $t$ through the single variable $z=x+c t$, with $c \geq 0$ without loss of generality (this gives a leftward moving wave). In terms of the variable $z$, system (4.6) becomes

$$
\begin{aligned}
c S^{\prime}(z)= & D_{s} S^{\prime \prime}(z)+\alpha S(z)\left(1-\frac{S(z)}{\gamma}\right)-K S(z) P(z) \\
c P^{\prime}(z)= & D_{p} P^{\prime \prime}(z)-\mu_{p} P(z)-m P^{2}(z)-K S(z) P(z) \\
& +b K e^{-\mu_{i} T} \int_{-\infty}^{\infty} \frac{e^{-y^{2} / 4 D_{i} T}}{2 \sqrt{\pi D_{i} T}} S(z-c T-y) P(z-c T-y) d y
\end{aligned}
$$

where prime denotes differentiation with respect to $z$, and we need to solve (4.7) for $S(z)$ and $P(z)$ subject to

$$
(S, P)(-\infty)=(\gamma, 0) \quad \text { and } \quad(S, P)(+\infty)=\left(S^{*}, P^{*}\right)
$$

System (4.7), (4.8) remains a difficult mathematical problem, and we have not been able to establish the existence of a solution, even with the most recently developed methods for proving existence of traveling front solutions of delay reaction-diffusion systems such as those of Wu and Zou [21]. We shall therefore assume that such a solution exists and concentrate on finding out as much as possible about the speed $c$ at which the virus infection spreads through the spatial domain. On the further assumption that the infection spreads at the minimum speed consistent with having an ecologically realistic solution satisfying $S(z), P(z) \geq 0$ for all $z \in(-\infty, \infty)$, we shall formally calculate this minimum speed by examining the situation as $z \rightarrow-\infty$, where $P(z) \rightarrow 0$, and obtaining conditions on $c$ which are necessary for the convergence of $P(z)$ to 0 to be nonoscillatory. Linearizing as $z \rightarrow-\infty$, when $P \rightarrow 0$ and $S \rightarrow \gamma$, the 
second equation of (4.7) becomes, approximately,

$c P^{\prime}(z)=D_{p} P^{\prime \prime}(z)-\mu_{p} P(z)-\gamma K P(z)+b \gamma K e^{-\mu_{i} T} \int_{-\infty}^{\infty} \frac{e^{-y^{2} / 4 D_{i} T}}{2 \sqrt{\pi D_{i} T}} P(z-c T-y) d y$

and has solutions of the form $P(z)=\exp (\lambda z)$ whenever $\lambda$ satisfies

$$
c \lambda-D_{p} \lambda^{2}+\mu_{p}+\gamma K=b \gamma K e^{-\mu_{i} T} e^{-\lambda c T} e^{\lambda^{2} D_{i} T} .
$$

Since this analysis is for $z \rightarrow-\infty$, it is necessary that (4.9) have at least one real positive root if $P(z)$ is to approach 0 in a nonoscillatory manner. Whether (4.9) has real positive roots or not depends on the value of $c$, as can be easily seen by plotting the leftand right-hand sides of (4.9) against $\lambda$ and remembering that $b \gamma K e^{-\mu_{i} T}>\mu_{p}+\gamma K$, since this is the condition for the existence of $\left(S^{*}, P^{*}\right)$. If $c$ is very small, then (4.9) has no real positive roots, but if $c$ is gradually increased, there is a critical value of $c$ which we shall call $c_{\min }$ (depending on $T$ ) such that when $c=c_{\min }$ (4.9) has one positive root (a double root), and when $c>c_{\min }$ the equation has precisely two real distinct positive roots. Only traveling fronts for which $c \geq c_{\min }$ are ecologically realistic, and we assume that the virus infection travels with speed $c_{\min }$ since it is usually the case in reaction-diffusion equations that the front one actually sees is the one with minimum speed (those with $c>c_{\min }$ usually have very small basins of attraction that rule out all but special initial conditions having very specific exponential decay rates).

Our aim now is to find out more about $c_{\min }$ and its dependence on the parameters. It is not possible to find an explicit expression for $c_{\min }$, but we can find some information about it. Indeed, $c_{\min }$ is the value of $c$ for which (4.9) has a double root $\lambda_{*}$. Therefore, $c_{\min }$ and the double root $\lambda_{*}$ must satisfy the simultaneous equations

$$
\begin{gathered}
c_{\min } \lambda_{*}-D_{p} \lambda_{*}^{2}+\mu_{p}+\gamma K=b \gamma K \exp \left(-\mu_{i} T-\lambda_{*} c_{\min } T+\lambda_{*}^{2} D_{i} T\right), \\
c_{\min }-2 D_{p} \lambda_{*}=b \gamma K\left(2 \lambda_{*} D_{i} T-c_{\min } T\right) \exp \left(-\mu_{i} T-\lambda_{*} c_{\min } T+\lambda_{*}^{2} D_{i} T\right) .
\end{gathered}
$$

From these equations, we see that $\lambda_{*}$ must satisfy $f(\lambda)=0$, where

$$
\begin{gathered}
f(\lambda):=2 D_{i} D_{p} T \lambda^{3}-\left(2 c_{\min } D_{i} T+c_{\min } D_{p} T\right) \lambda^{2}-\left(2 D_{i} T\left(\mu_{p}+\gamma K\right)-c_{\min }^{2} T+2 D_{p}\right) \lambda \\
+c_{\min }+c_{\min } T\left(\mu_{p}+\gamma K\right) .
\end{gathered}
$$

Now $f$ is a cubic and is such that $f(0)>0$ and

$$
f\left(\frac{c_{\min }+\sqrt{c_{\min }^{2}+4 D_{p}\left(\mu_{p}+\gamma K\right)}}{2 D_{p}}\right)=-\sqrt{c_{\min }^{2}+4 D_{p}\left(\mu_{p}+\gamma K\right)}<0 .
$$

These facts imply that the equation $f(\lambda)=0$ has one real negative root and two real distinct positive roots. The larger of the two positive roots cannot satisfy the first equation of (4.10). Therefore, $\lambda_{*}$ is the smaller of the two real positive roots of $f(\lambda)=0$. Furthermore,

$$
0<\lambda_{*}<\frac{c_{\min }+\sqrt{c_{\min }^{2}+4 D_{p}\left(\mu_{p}+\gamma K\right)}}{2 D_{p}} .
$$

The roots of a cubic equation are difficult to write down in general terms because there are numerous cases depending on the signs of various quantities defined in terms of the coefficients in the equation. An appendix to the book by Murray [15] gives all the 
details. Although the coefficients of our particular cubic equation are complicated, we know a priori that our cubic equation has only real roots, and this narrows down the possibilities considerably. In fact, if we let

$$
\begin{gathered}
a_{*}=-\frac{c_{\min }\left(2 D_{i}+D_{p}\right)}{6 D_{i} D_{p}}, \\
\alpha_{*}=\frac{4 c_{\min }^{2} T D_{i}^{2}-2 c_{\min }^{2} T D_{i} D_{p}+c_{\min }^{2} T D_{p}^{2}+12 D_{i}^{2} D_{p} T \mu_{p}+12 D_{i}^{2} D_{p} T \gamma K+12 D_{i} D_{p}^{2}}{36 D_{i}^{2} D_{p}^{2} T}
\end{gathered}
$$

(it is easily shown that $\alpha_{*}>0$ ),

$$
\begin{gathered}
N=8 c_{\min }^{2} T D_{i}^{2}+36 D_{i}^{2} D_{p} T \mu_{p}+36 D_{i}^{2} D_{p} T \gamma K+2 c_{\min }^{2} T D_{i} D_{p}-18 D_{i} D_{p}^{2}-c_{\min }^{2} T D_{p}^{2}, \\
\beta_{*}=\frac{c_{\min }\left(D_{p}-D_{i}\right) N}{108 D_{i}{ }^{3} D_{p}{ }^{3} T},
\end{gathered}
$$

and

$$
\phi=(1 / 3) \sin ^{-1}\left(\frac{\beta_{*}}{2 \alpha_{*}^{3 / 2}}\right), \quad \phi \in[-\pi / 6, \pi / 6],
$$

then the only root of $f(\lambda)=0$ satisfying (4.11) can be shown to be

$$
\lambda_{*}=2 \alpha_{*}^{1 / 2} \sin \phi-a_{*} .
$$

Substituting $\lambda_{*}$ into either equation of (4.10) then gives a single, but very complicated, equation determining the speed $c_{\min }$.

We define the function $g(c)$ to be the left-hand side minus the right-hand side of the second equation of (4.10), with $\lambda_{*}$ given by (4.12) and $c_{\min }$ replaced by $c$. The resulting function is too complicated to write out explicitly but is easily handled in MAPLE. Of course, $c_{\min }$ solves $g\left(c_{\min }\right)=0$ and can easily be found either by reading off the root from an accurate plot of $g(c)$ or by using MAPLE commands for finding roots numerically. Figure 3 shows a plot of $g(c)$ for typical parameter values (see caption). We investigated how $c_{\text {min }}$ depends on the values of all the parameters, and our main observations were as follows:

- If $\mu_{i}, \mu_{p}$, or $T$ is increased, the result is a decrease in $c_{\mathrm{min}}$.

- If $K, \gamma, b, D_{i}$, or $D_{p}$ is increased, the result is an increase in $c_{\min }$.

- If the delay $T$ is large, then the value of $c_{\min }$ is much more sensitive to $D_{i}$ than to $D_{p}$. Presumably this is because virus particles with a host are transported at the diffusivity of the infectives. To illustrate this, let $T=7$ and other parameters retain their Figure 3 values. Then $c_{\min }=1.265$. Keeping $T=7$, if $D_{i}$ is then raised to $100, c_{\text {min }}$ rises to 5.623. But if instead $D_{i}=5$ and $D_{p}$ is raised to 100 , then $c_{\text {min }}$ rises only to 1.976 .

Analytical estimates for $c_{\min }$ can be obtained from other arguments, involving consideration of the graphs of the left- and right-hand sides of (4.9) as functions of $\lambda$. When $c=c_{\min }$ these two graphs just touch, at the value $\lambda_{*}$ just discussed. Consider first the case when $D_{i}<D_{p}$ (so the minimum of the right-hand side is to the right of the maximum of the left-hand side). In this situation the maximum of the left-hand 


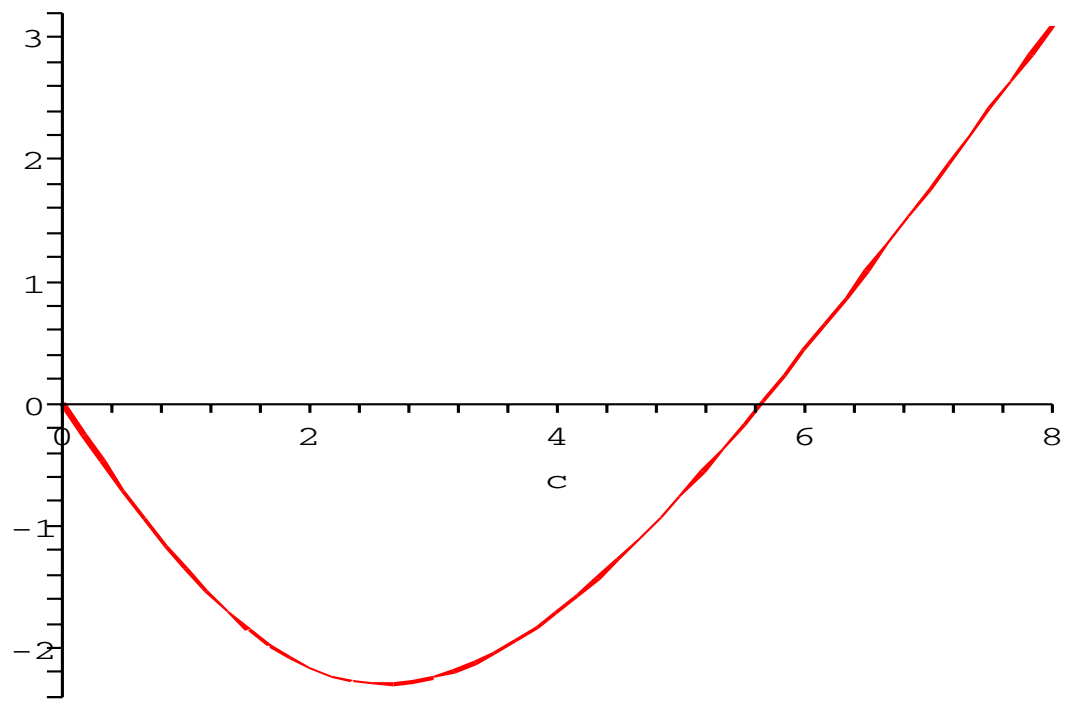

FIG. 3. Plot of the function $g(c)$ defined in the text. The virus is predicted to spread at the speed $c_{\text {min }}>0$ such that $g\left(c_{\min }\right)=0$. Parameter values used for this graph were $K=0.134, \mu_{i}=0.1$, $\mu_{p}=2, T=0.2, \gamma=1, D_{i}=5, D_{p}=1$, and $b=60$. For these values, $c_{\min }=5.646$.

side as a function of $\lambda$ is $c_{\min }^{2} /\left(4 D_{p}\right)+\mu_{p}+\gamma K$, and this must be less than the value of the right-hand side when $\lambda=0$, which is $b \gamma K e^{-\mu_{i} T}$. This leads to the estimate

$$
c_{\min }<2 \sqrt{D_{p}\left\{\gamma K\left(b e^{-\mu_{i} T}-1\right)-\mu_{p}\right\}} \text { if } D_{i}<D_{p} .
$$

If $D_{i}$ is larger than $D_{p}$, but not too much larger, the above estimate on $c_{\min }$ will still hold.

We also carried out some numerical simulations of system (4.6) with a view to finding out whether the minimum speed $c_{\text {min }}$ found from the linearized analysis is the speed which would be observed in practice. The question is whether the minimum speed wave is in some sense robust, attracting large classes of initial data. These questions are difficult to resolve analytically. In a recent paper, Thieme and Zhao [19] proved results on asymptotic speeds of spread for a class of nonlinear integral equations which include many reaction-diffusion models with delay, but their results do not include system (4.6). Figure 4 shows the results of a numerical simulation of system (4.6). For initial data, susceptibles $S$ were set equal to $\gamma$ throughout the domain, and some phages were introduced at $x=0$ into an otherwise phage-free domain. Figure 4 shows how the phages spread out into the domain and the effect on the density of susceptible bacteria. Note that the traveling wave profiles are nonmonotone. Careful examination of the profiles suggests that the traveling fronts advance at the minimum speed $c_{\min }$ computed from the linearized analysis. The numerically computed front actually appears to travel at a slightly higher speed, but we are confident that this is purely a consequence of the discretization procedure. The speed varied slightly with the number of spatial grid points but seemed to approach $c_{\min }$ from above as 

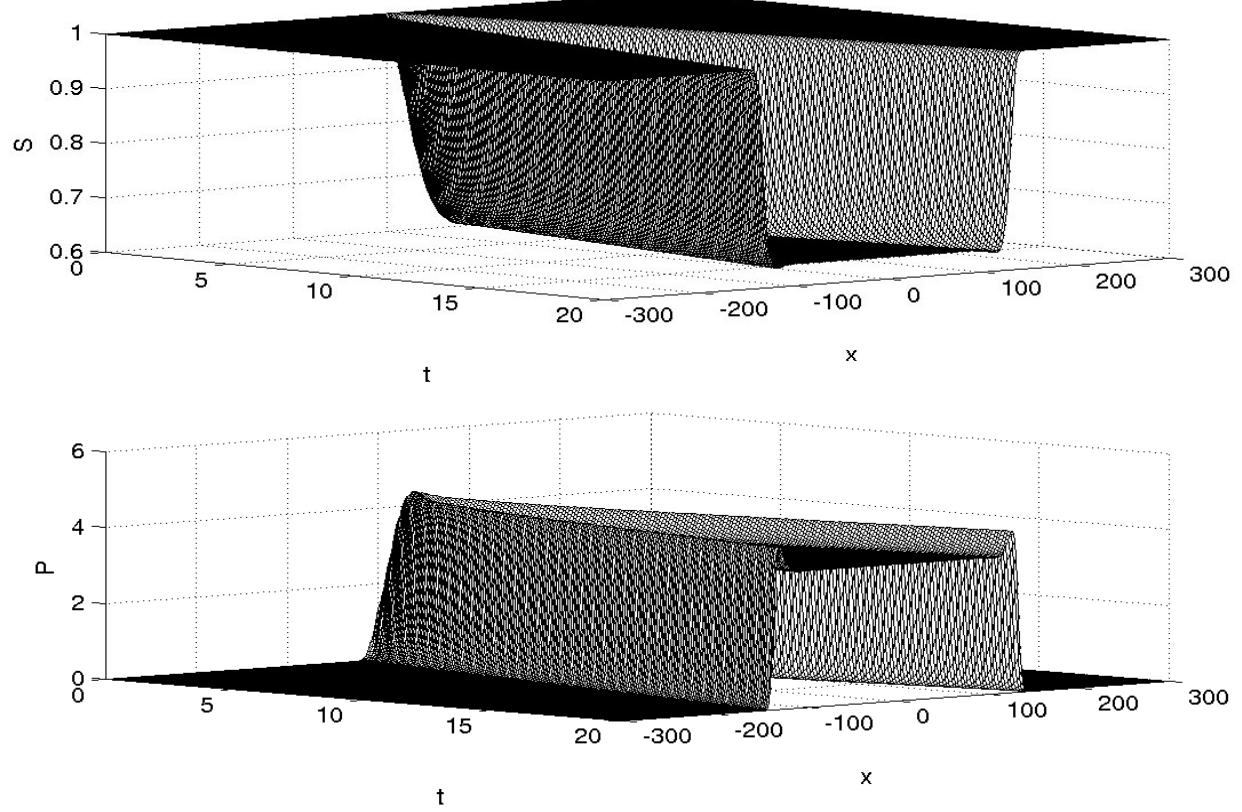

FIG. 4. Numerical simulation of system (4.6). Parameter values were $D_{s}=5, m=1, \alpha=1.34$, and the remaining parameters were as in Figure 3. The simulation suggests that the asymptotic speed of spread is $c_{\min }$, the minimum speed according to the linearized analysis.

the number of grid points was increased. As a result, we suggest that the asymptotic speed of spread is indeed the speed $c_{\text {min }}$ found from the linearized analysis.

5. Discussion. A key observation of Beretta and Kuang $[2,4]$ is the sensitivity of the dynamics on the phage reproduction rate $b$. This remains so for model (2.1). The novel observation of this work is the ultrasensitivity of the dynamics on the phage density dependent mortality rate $m$. This suggests that the density dependent mortality rate must be carefully measured to gain a better understanding of the bacteriophage infection dynamics in marine bacteria. Indeed, the recent work of Kuang, Fagan, and Loladze [13] contends that the predator death rate almost always positively correlates with the predator density in nature. To see this for model (2.1), we present Figures 5 and 6 . Both figures use initial data and parameter values identical to those in Figure 2, except that in Figure 5, $m=0$, while in Figure $6, m=0.2$.

The second novel aspect of our work is the rigorous derivation of a delay reactiondiffusion system to model the spatial spread of the virus infection and the use of this system to formally calculate the speed at which the infection spreads through a one-dimensional environment. The speed does not depend on the density dependent mortality parameter $m$ just discussed. Unfortunately, it is not possible to find a simple expression for the speed, but it can be found from numerical computation. As we would expect, the speed depends on the diffusivity of both the infectives and the phages but is much more sensitive to the value of the former than the latter. This would be because virus replication takes place only inside a host, and therefore during replication the diffusivity of the viruses is effectively the host diffusivity $D_{i}$ rather than $D_{p}$. 

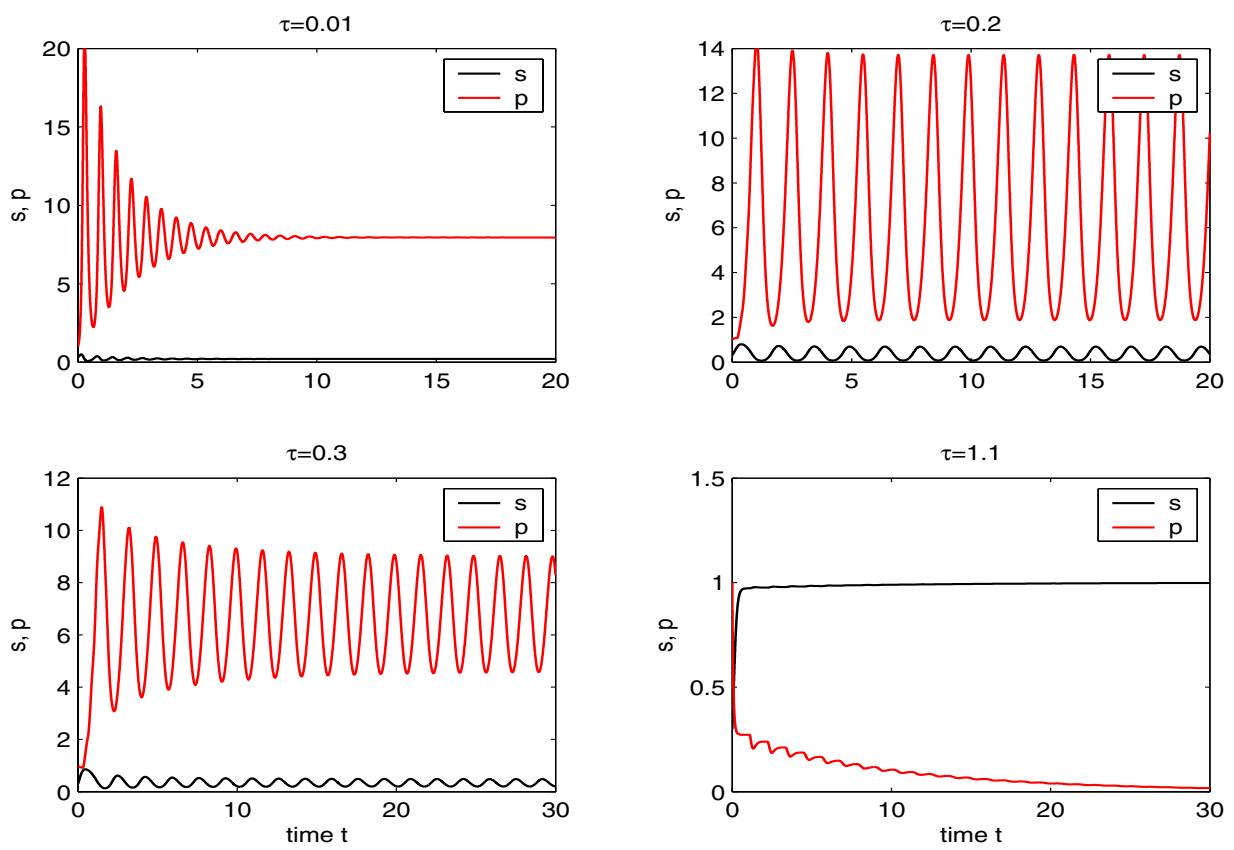

FIG. 5. A solution of model (3.16) with $s(\theta)=0.3, p(\theta)=1, \theta \in[-\tau, 0]$, where $\mu_{p}=14.925$, $b=75, \mu_{i}=1.5, \alpha=10, m=0$, and $\tau$ varies from 0.01 to 1.1 .
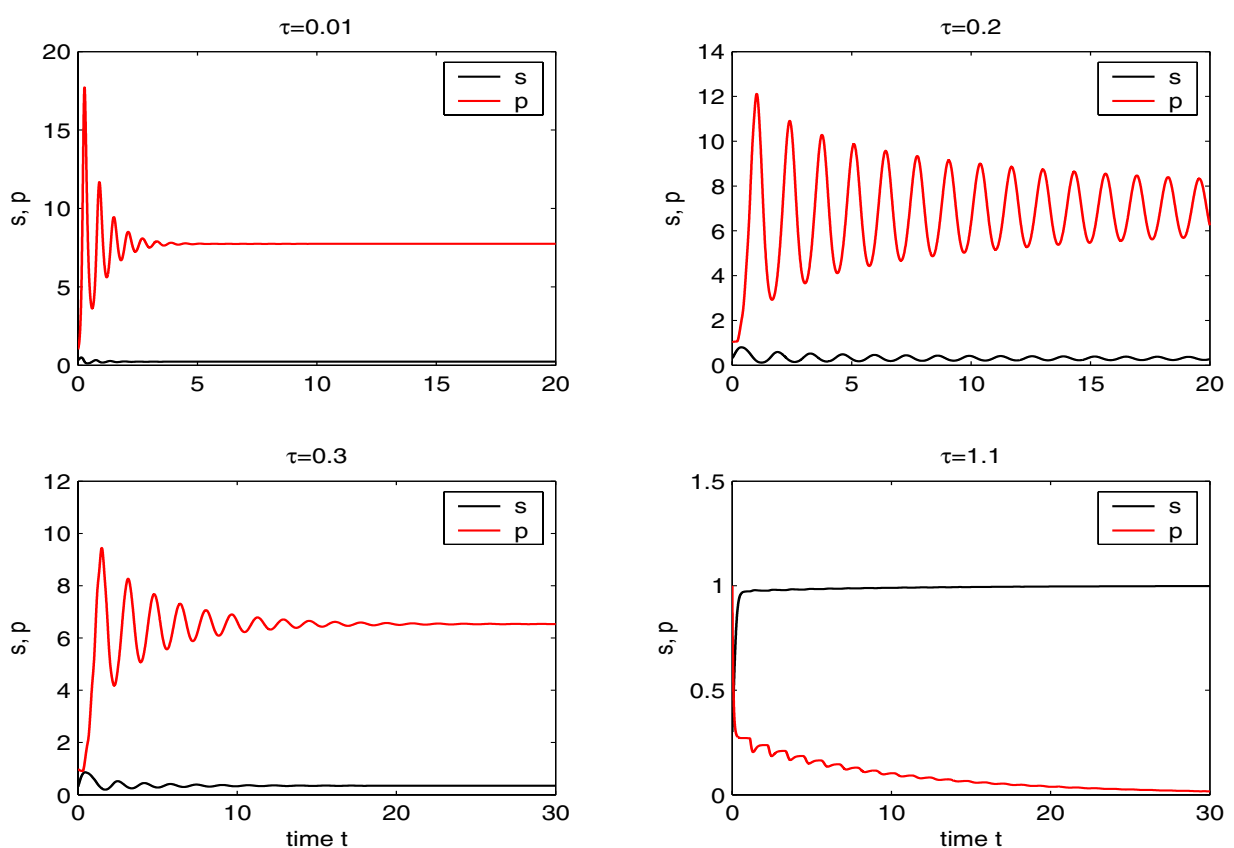

FIG. 6. A solution of model (3.16) with $s(\theta)=0.3, p(\theta)=1, \theta \in[-\tau, 0]$, where $\mu_{p}=14.925$, $b=75, \mu_{i}=1.5, \alpha=10, m=0.2$, and $\tau$ varies from 0.01 to 1.1 . 
Acknowledgment. We would like to thank Zdzislaw Jackiewicz of Arizona State University for help with the numerical simulation of the reaction-diffusion model.

\section{REFERENCES}

[1] E. Beltrami and T. O. Carroll, Modeling the role of viral disease in recurrent phytoplankton blooms, J. Math. Biol., 32 (1994), pp. 857-863.

[2] E. Beretta AND Y. KuANG, Modeling and analysis of a marine bacteriophage infection, Math. Biosci., 149 (1998), pp. 57-76.

[3] E. Beretta, M. Carletti, And F. Solimano, On the effects of environmental fluctuations in a simple model of bacteria-bacteriophage infection, Canad. Appl. Math. Quart., 8 (2000), pp. 321-366.

[4] E. Beretta And Y. KuAng, Modeling and analysis of a marine bacteriophage infection with latency period, Nonlinear Anal. Real World Appl., 2 (2001), pp. 35-74.

[5] E. Beretta And Y. Kuang, Geometric stability switch criteria in delay differential systems with delay dependent parameters, SIAM. J. Math. Anal., 33 (2002), pp. 1144-1165.

[6] O. Bergh, K.Y. Borsheim, G. Bratbak, and M. Heldal, High abundance of viruses found in aquatic environments, Nature, 340 (1989), pp. 467-468.

[7] B. J. M. Bohannan And R. E. Lenski, Linking genetic change to community evolution: Insights from studies of bacteria and bacteriophage, Ecology Letters, 3 (2000), pp. 362-377.

[8] A. Campbell, Conditions for the existence of bacteriophage, Evolution, 15 (1961), pp. 153-165.

[9] M. Carletti, Numerical determination of the instability region for a delay model of phagebacteria interaction, Numer. Algorithms, 28 (2001), pp. 27-44.

[10] M. CARLETTI, On the stability properties of a stochastic model for phage-bacteria interaction in open marine environment, Math. Biosci., 175 (2002), pp. 117-131.

[11] S. A. GOURLEY, AND J. W.-H. So, Extinction and wavefront propagation in a reaction-diffusion model of a structured population with distributed maturation delay, Proc. Roy. Soc. Edinburgh Sect. A, 133 (2003), pp. 527-548.

[12] Y. Kuang, Delay Differential Equations, with Applications in Population Dynamics, Academic Press, Boston, 1993.

[13] Y. Kuang, W. F. Fagan, and I. Loladze, Biodiversity, habitat area, resource growth rate and interference competition, Bull. Math. Biol., 65 (2003), pp. 497-518.

[14] R. E. Lenski AND B. R. Levin, Constraints on the coevolution of bacteria and virulent phage: A model, some experiments, and predictions for natural communities, Amer. Naturalist, 125 (1985), pp. 585-602.

[15] J. D. Murray, Mathematical Biology, Springer-Verlag, Berlin, 1993.

[16] L. M. Proctor and J. A. Fuhrman, Viral mortality of marine bacteria and cyanobacteria, Nature, 343 (1990), pp. 60-62.

[17] H. L. Smith, A structured population model and a related functional-differential equation: Global attractors and uniform persistence, J. Dynam. Differential Equations, 6 (1994), pp. $71-99$.

[18] J. W.-H. So, J. Wu, AND X. Zou, A reaction-diffusion model for a single species with age structure. I. Travelling wavefronts on unbounded domains, Proc. Roy. Soc. London Ser. A, 457 (2001), pp. 1841-1853.

[19] H. R. Thieme AND X.-Q. ZhaO, Asymptotic speeds of spread and traveling waves for integral equations and delayed reaction-diffusion models, J. Differential Equations, 195 (2003), pp. $430-470$.

[20] G. S. K. Wolkowicz, H. XIA, and J. WU, Global dynamics of a chemostat competition model with distributed delay, J. Math. Biol., 38 (1999), pp. 285-316.

[21] J. Wu AND X. Zou, Travelling wave fronts of reaction-diffusion systems with delay, J. Dynam. Differential Equations, 13 (2001), pp. 651-687. 